\title{
Loss of Fhit Expression in Testicular Germ Cell Tumors and Intratubular Germ Cell Neoplasia
}

\author{
Eduardo Eyzaguirre, M.D., Zoran Gatalica, M.D., D.Sc. \\ Department of Pathology, Division of Surgical Pathology, The University of Texas Medical Branch, \\ Galveston, Texas
}

The FHIT gene, located at human chromosome 3p14.2, is frequently deleted in a number of human cancers, and interstitial deletions at this site were recently described in a significant proportion $(41 \%)$ of testicular germ cell tumors. We studied the expression of Fhit protein in the progression and differentiation of testicular germ cell tumors to further elucidate its role in this type of malignancy. Fortyfive patients with testicular germ cell tumors and intratubular germ cell neoplasia (identified in 42/45 cases) were included in the study. Immunohistochemical staining with polyclonal rabbit IgG antibody to Fhit (ZR44, Zymed Laboratories) on formalin-fixed, paraffin-embedded tissues was used. Fhit was constitutively expressed in germ cells, Sertoli cells, and Leydig cells. All 42 cases of intratubular germ cell neoplasia revealed no expression of this protein. No expression of Fhit was observed in any case of pure seminoma or in the seminomatous component of mixed germ cell tumors. Unexpectedly, Fhit expression was frequently $(16 / 18)$ observed in the glandular tissue of mature teratomatous component of mixed germ cell tumors, despite the absence of Fhit in the intratubular germ cell neoplasia, the presumed precursor lesion. The loss of Fhit expression is a consistent characteristic of intratubular germ cell neoplasia, which suggests a potential role in a maturation/differentiation defect early in the development of testicular germ cell tumors. Likewise, the lack of expression in seminomas is supportive of this view. However, reexpression of Fhit in well-differentiated glandular epithelium of teratomatous component of mixed germ cell tumors suggests that there is no loss of

\footnotetext{
Copyright () 2002 by The United States and Canadian Academy of Pathology, Inc.

VOL. 15, NO. 10, P. 1068, 2002 Printed in the U.S.A.

Date of acceptance: June 21, 2002.

The results of this study were presented in part at the 89th USCAP Annual Meeting, March 2000.

Address reprint requests to: Zoran Gatalica, M.D., D.Sc., Department of Pathology, Creighton University Medical Center, 601 N. 30th St., Omaha, NE 68131; e-mail: zgatalica@pathology.creighton.edu.

DOI: 10.1097/01.MP.0000028574.10294.7D
}

FHIT gene in this subset of neoplasia but rather that Fhit protein expression is differently regulated through the phases of germ cell tumor progression.

KEY WORDS: Cancer, Fhit, Immunohistochemistry, Testicular neoplasms.

Mod Pathol 2002;15(10):1068-1072

Testicular tumors are the most common malignancies of adult and adolescent males 14-40 years old $(1,2)$, and the incidence of such tumors has increased substantially from 1973 to 1996 in North America $(2,3)$. Ninety percent of testicular tumors arise from germ cells. Testicular germ cell tumors are a heterogeneous group of neoplasms classified into seminomas and nonseminomas. It is generally accepted that testicular germ cell tumors (with the exception of spermatocytic seminomas) originate from a common precursor lesion known as intratubular germ cell neoplasia (1, 4-6). Cytogenetic and molecular studies have demonstrated that testicular germ cell tumors and intratubular germ cell neoplasia consistently exhibit increased copies of chromosome $12 \mathrm{p}$ material (1, 7-9). In addition, molecular analysis using variable number of tandemrepeats markers and comparative genomic hybridization techniques have repeatedly identified loss of heterozygosity at chromosome $3 p$ in testicular germ cell tumors, a finding that suggests the presence of one or more tumor suppressor genes at this region (10). Although molecular pathology studies have demonstrated the alteration of protooncogenes (c-Kit), tumor suppressor genes (p53, DCC, and pRb), as well as aberrant expression of the cyclin D2 gene, there are as of yet no specific tumor suppressor genes associated with the histopathologic development of testicular germ cell tumors. Moreover the fundamental genetic factors that play a role in the development and progression of testicular germ cell tumors are not yet well understood.

The fragile histidine triad (FHIT) tumor suppressor gene located in the fragile locus FRA3B at chromosome 3p14.2 has been frequently involved in 
homozygous deletions and genetic rearrangement in a variety of sporadic human tumors (11-15). The product of this gene, Fhit protein, is a diadenosine oligophosphate hydrolase capable of altering cell cycle kinetics and/or inducing apoptosis (16-21). Alterations in the FHIT locus have been associated with reduction or loss of Fhit protein expression in malignant and premalignant lesions including carcinomas of lung, cervix, kidney, esophagus, breast, and other organs (22). More recently, suppression of tumor growth in nude mice injected with tumor cells transfected with FHIT provide direct evidence that FHIT is a tumor suppressor gene $(23,24)$. To further elucidate the role of Fhit protein in the development of testicular germ cell tumors, we studied the expression of Fhit protein in a series of testicular germ cell tumors and their precursor lesion intratubular germ cell neoplasia and compared it with the expression in normal epithelium of seminiferous tubules.

\section{MATERIAL AND METHODS}

\section{Tissues}

Formalin-fixed, paraffin-embedded tissues from 45 patients with testicular germ cell tumors were used in the study. Intratubular germ cell neoplasia was identified in the surrounding tubules in 42 cases by the presence of undifferentiated, atypical, enlarged germ cells with clear cytoplasm and strong membranous staining with antibodies against placental alkaline phosphatase. Histologically, testicular germ cell tumors were diagnosed as follows: seminomas (16 cases), pure embryonal carcinomas ( 2 cases), pure yolk sac tumor (2 cases), pure choriocarcinoma ( 1 case), and mixed germ cell tumors (24 cases). The histologic tumor types encountered in mixed germ cell tumors included combinations of: seminoma and embryonal carcinoma (4 cases); seminoma, embryonal carcinoma and teratoma (1 case); embryonal carcinoma and teratoma (7 cases); yolk sac tumor, embryonal carcinoma and teratoma (9 cases); yolk sac tumor and teratoma (2 cases); and yolk sac tumor and embryonal carcinoma (1 case).

\section{Immunohistochemical Studies}

Polyclonal rabbit IgG antibody to Fhit (ZR44, Zymed Laboratories, Inc., San Francisco, CA) was used at 1:200 dilution. Placental alkaline phosphatase immunostaining was performed using a mouse monoclonal antibody (clone 8A9; Neomarkers, Inc., Union City, CA). The slides were deparaffinized and subjected to heat-induced epitope retrieval using a steamer (Black and Decker Handy Steamer Plus) and DAKO Target retrieval solution $(\mathrm{pH}$ 6.0-6.2;
DAKO Corp., Carpinteria, CA). The slides were steamed for 20 minutes, cooled at room temperature for 20 minutes, rinsed with deionized water, and then placed in Tris-buffered saline for $5 \mathrm{~min}$ utes. Endogenous peroxidase was blocked by placing the slides in $3 \%$ hydrogen peroxide for $10 \mathrm{~min}$ utes. Immunohistochemical staining was performed using the automated immunostainer (DAKO). A standard streptavidin-biotin complex technique (LSAB2 system, DAKO) with diaminobenzidine as chromogen was used. The slides were counterstained with hematoxylin (13). Expression of Fhit was classified as follows: absent/weak (cytoplasmic staining less intense than in adjacent normal germ cells) and strong (staining of similar intensity to that of normal germ cells). The extent of positive staining was divided into the following categories: focal ( $<10 \%$ positive tumor cells), moderate $(10-50 \%$ positive tumor cells), and diffuse ( $>50 \%$ positive tumor cells).

\section{RESULTS}

\section{Expression of Fhit in the Normal Testis}

In the normal testicular parenchyma, Fhit expression was uniformly strong in the cytoplasm of germ cells and Sertoli cells, whereas Leydig cells variably expressed Fhit (Fig. 1A).

\section{Expression of Fhit and Placental Alkaline \\ Phosphatase in Intratubular Germ Cell Neoplasia}

Intratubular germ cell neoplasia was identified within the seminiferous tubules surrounding the germ cell tumors of 42 cases. The intratubular neoplastic cells were invariably negative for Fhit (Fig. 1A). In contrast, all cases of intratubular germ cell neoplasia were positive for placental alkaline phosphatase, confirming their neoplastic origin (inset of Fig. 1A).

\section{Expression of Fhit and Placental Alkaline Phosphatase in Invasive Germ Cell Tumors}

There was no detectable cytoplasmic immunostaining for Fhit protein in any of the malignant cells of 22 seminomas, 23/24 embryonal carcinomas, 14/14 yolk sac tumors, and 7/10 immature teratomas (Table 1; Fig. 1, B-D). One case of embryonal carcinoma and three cases of immature teratoma showed focal weak cytoplasmic staining for Fhit protein. In contrast, the majority of mixed germ cell tumors (88\%) strongly expressed cytoplasmic Fhit protein primarily in the most differentiated elements (e.g., mature glandular epithelium of teratomas), whereas immature germ cell components (embryonal carcinoma and yolk sac elements) did not stain (Fig. 1, E-F). 


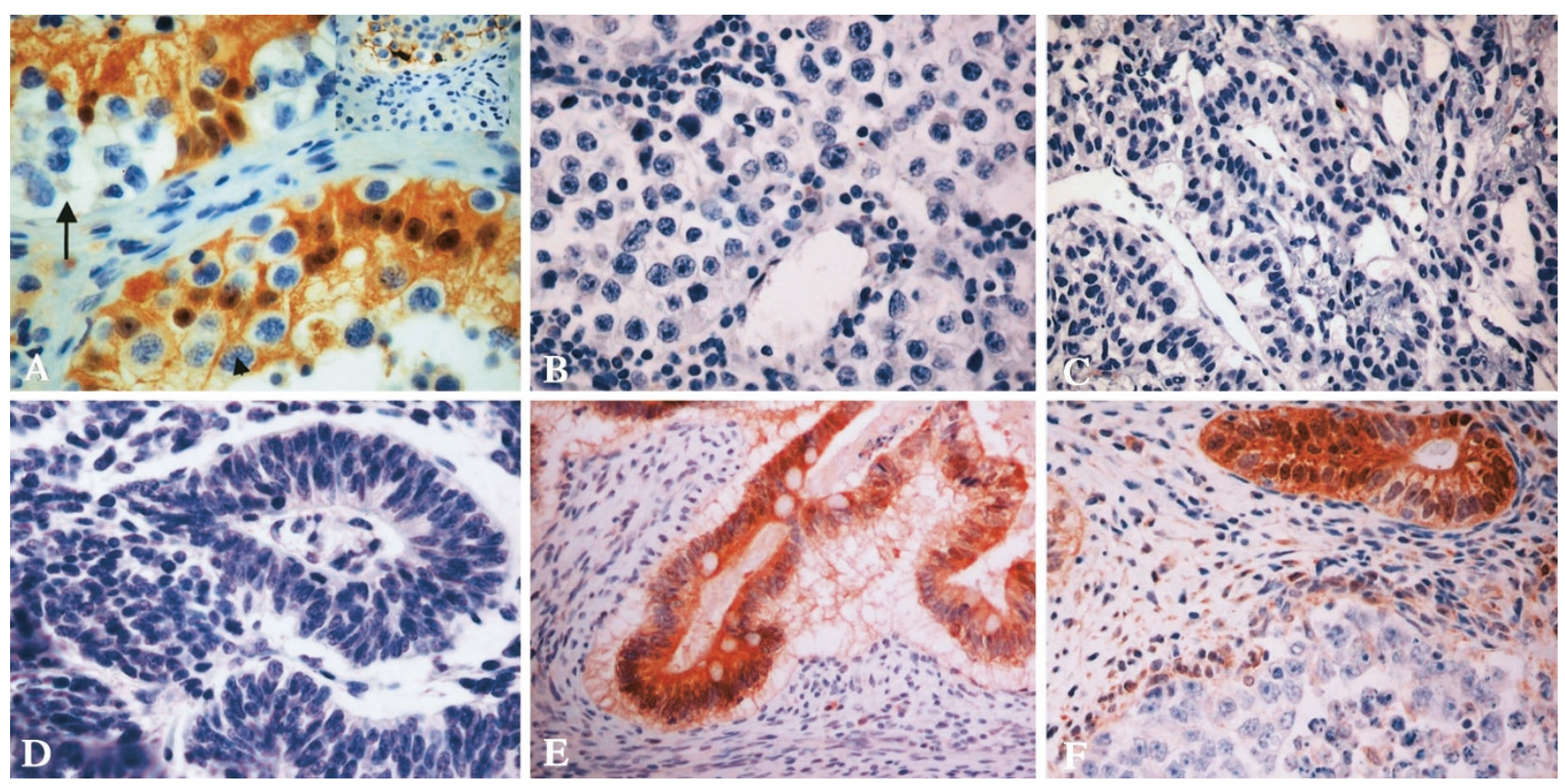

FIGURE 1. Immunohistochemical detection of Fhit protein expression in ITGCN and TGCT. A, loss of Fhit expression in ITGCN (arrow), and strong Fhit expression in normal germ cells (arrowhead), Sertoli cells and Leydig cells. Inset, antibody against placental alkaline phosphatase highlights intratubular neoplastic germ cells. B, seminoma lacking expression of Fhit protein. C \& D, Fhit-negative yolk sac tumor and immature teratoma, respectively. E, strong Fhit expression in the cytoplasm of epithelial elements of a mature teratoma. F, teratocarcinoma showed strong expression of Fhit in mature teratomatous epithelium and no expression in embryonal carcinoma elements (all slides show immunohistochemical stain with polyclonal rabbit anti-Fhit antibody and 3,3'-diaminobenzidine as a chromogen, $400 \times$ ).

TABLE 1. Fhit Expression in Human Testicular Germ Cell Tumors and Intratubular Germ Cell Neoplasia

\begin{tabular}{|c|c|c|c|c|}
\hline \multirow{2}{*}{ Tumor Type } & \multicolumn{3}{|c|}{ Fhit Reactivity } & \multirow{2}{*}{$\%$ Negative } \\
\hline & Positive & Negative & Total & \\
\hline $\begin{array}{l}\text { Intratubular germ cell } \\
\text { neoplasia }\end{array}$ & 0 & 42 & 42 & 100 \\
\hline Seminomas & 0 & 17 & 17 & 100 \\
\hline $\begin{array}{l}\text { Seminomas in mixed } \\
\text { germ cell tumors }\end{array}$ & 0 & 5 & 5 & 100 \\
\hline Embryonal carcinomas ${ }^{a}$ & 1 & 23 & 24 & 96 \\
\hline Mature teratomas $^{a}$ & 16 & 2 & 18 & 12.5 \\
\hline Immature teratomas $^{a}$ & 3 & 7 & 10 & 70 \\
\hline Yolk sac tumor ${ }^{a}$ & 0 & 14 & 14 & 100 \\
\hline Choriocarcinomas & 0 & 1 & 1 & 100 \\
\hline
\end{tabular}

${ }^{a}$ Includes 2 pure embryonal carcinomas, 22 embryonal carcinomas in mixed germ cell tumors, 2 pure yolk sac, and 12 yolk sac tumors in mixed germ cell tumors; all teratomas occurred in mixed germ cell tumors.

Placental alkaline phosphatase was detectable by immunohistochemistry in 19/22 (86\%) seminomas, $2 / 14$ (14.3\%) yolk sac tumors, and in 7/24 (29\%) embryonal carcinomas (not shown).

\section{DISCUSSION}

The putative tumor suppressor gene FHIT is located at chromosome 3p14.2 and contains the most active of the common human chromosomal fragile regions, FRA3B. Alterations of the FHIT gene and loss of Fhit protein expression have been identified across a variety of human cancers and in premalig- nant lesions of lung, colon, breast, and cervix (14, $15,22,25)$.

Neoplastic disease progression, from normal to premalignant to malignant phenotypes, is associated with genetic instability manifested by alteration of gene expression that is often associated with characteristic morphologic phenotypes (26). It is generally well accepted that most but not all testicular germ cell tumors arise from a common neoplastic precursor lesion, intratubular germ cell neoplasia. According to this model seminomas evolve directly from intratubular germ cell neoplasia and embryonal carcinomas could arise directly from intratubular germ cell neoplasia or through an intermediate stage corresponding to seminoma. The other types of testicular germ cell tumors, teratomas, yolk sac tumors, and choriocarcinomas, may evolve directly from seminoma or embryonal carcinoma (5-8). This concept is supported by karyotype analyses that demonstrated a marker chromosome, i(12p) in seminomas and nonseminomatous germ cell tumors, as well as in intratubular germ cell neoplasia $(1,5-8)$. Moreover, the study of Lothe et al. (10) and more recently that of Kraggerud et al. (27), demonstrated that testicular germ cell tumors commonly showed loss of heterozygosity at $3 p$ and $11 p$ chromosomes, further suggesting that these regions may contain candidate tumor suppressor genes important in pathogenesis of these testicular neoplasms and that FHIT is one of them. 
In our study, we found loss of Fhit protein expression in all cases of pure seminoma or the seminomatous component of mixed germ cell tumors, $96 \%$ cases of embryonal carcinomas, $70 \%$ cases of immature teratomas and all yolk sac tumors. Additionally, Fhit protein expression was absent in all cases of intratubular germ cell neoplasia adjacent to invasive germ cell tumors. Similarly, Kraggerud et al. (27) found loss of Fhit expression in two cases of intratubular germ cell neoplasia associated with nonseminomatous testicular germ cell tumors and a similar absence or reduction of Fhit expression between nonseminomatous and seminomatous tumors. These observations suggest that alterations of FHIT gene may play an important role early in the carcinogenesis of testicular germ cell tumors.

Whereas the function of FHIT as a tumor suppressor gene has been supported by experiments in cell cultures and more recently in Fhit-deficient mice $(19-21,23)$, the precise mechanism of Fhit protein action has yet to be determined. Recent data suggest that the tumor suppressor activity of FHIT could be related to regulation of apoptosis and the cell cycle through Fhit protein binding to Ap3A and alteration of the Ap4A/Ap3A ratio (16, 20, 28). Another study demonstrates that Fhit protein promotes assembly of microtubules through a specific binding of tubulin in the presence of microtubule associated proteins (29). These findings suggest that Fhit protein may exert its growth suppression activity interacting with microtubules and altering cell division.

More recent observations suggest that Fhit expression can inhibit tumor cell growth in vitro and in vivo through a caspase-dependent apoptosis pathway $(17,19,22)$. In a recent study, Roz et al. (30) found that adenoviral-mediated FHIT gene transfer into Fhit-negative lung and cervical cancer cell lines induced caspase-8-mediated apoptosis and suppression of in vivo tumorigenicity. Apoptosis is an essential element of normal spermatogenesis and alterations in the regulation of cell cycle and apoptosis undoubtedly play a role in the development of testicular germ cell tumors. Of interest is the finding that in the majority of mixed germ cell tumors, Fhit protein is absent among the less differentiated elements such as embryonal carcinoma and yolk sac tumor components but was present in the most differentiated areas such as glandular teratomatous epithelium. Re-expression of Fhit protein in the most differentiated teratomatous components of mixed germ cell tumors suggests that there is no loss or structural alteration of FHIT gene in these tumors but rather that Fhit expression is differently regulated (possibly silenced through promoter methylation; 31 ) through phases of germ cell tumor progression. Additionally, alternative splicing of FHIT mRNA has been frequently identi- fied in various neoplasms $(32,33)$, including testicular germ cell tumors (27). However, the significance of this finding is difficult to interpret because of the significant cellular heterogeneity of solid tumors and the lack of correlation between aberrant transcripts and protein expression levels.

In conclusion, this study demonstrates that loss of or decreased expression of Fhit protein is associated with the majority of testicular germ cell tumors. The loss of Fhit expression in adjacent intratubular germ cell neoplasia indicates that FHIT gene alterations play an important role in the growth control of germ cells and therefore in the genesis of testicular germ cell tumors. Moreover, the FHIT gene may represent a candidate target for early gene therapy management of patients with testicular germ cell tumors (22).

\section{REFERENCES}

1. Murty VVVS, Chaganti RSK. A genetic perspective of male germ cell tumors. Semin Oncol 1998;25:133-44.

2. Liu SL, Semenciw R, Waters C, Wen SW, Mery LS, Mao Y. Clues to the aetiological heterogeneity of testicular seminoma and non-seminomas: time trends and age-periodcohort effects. Int J Epidemiol 2000;29:826-31.

3. Jemal A, Thomas A, Murray T, Thum M. Cancer statistics, 2002. CA Cancer J Clin 2002;52:23-47.

4. Dieckmann KP, Skakkebaek NE. Carcinoma in situ of the testis: review of biological and clinical features. Int J Cancer 1999;83:815-22.

5. Chaganti RSK, Houldsworth J. Genetics and biology of adult human male germ cell tumors. Cancer Res 2000;60:1475-82.

6. Damjanov I. Pathogenesis of testicular germ cell tumors. Eur Urol 1993;23:2-7.

7. Geurts van Kessel A, Suijkerbuijk RF, Sinke RJ, Looijenga L, Oosterhuis JW, de Jong B. Molecular cytogenetics of human germ cell tumors: $\mathrm{i}(12 \mathrm{p})$ and related chromosomal anomalies. Eur Urol 1993;23:23-9.

8. Oosterhuis JW, Looijenga LHJ, Geurts van Kessel A, de Jong BA. Cytogenetic classification of germ cell tumors, and its biological relevance. Eur Urol 1993;23(Suppl 2):6-8.

9. Oliver RTD. Current opinion in germ cell cancer 2000. Curr Opin Oncol 200;12:249-54.

10. Lothe RA, Fosså SD, Stenwig AE, Nakamura Y, White R, Børresen AL, et al. Loss of 3p or 11p alleles is associated with testicular cancer tumors. Genomics 1989;5:134-8.

11. Campiglio M, Pekarsky Y, Menard S, Tagliabue E, Pilotti S, Croce CM. FHIT loss of function in human primary breast cancer correlates with advanced stage of the disease. Cancer Res 1999;59:3866-9.

12. Noguchi T, Muller W, Wirtz HC, Willers R, Gabbert HE. FHIT gene in gastric cancer: association with tumor progression and prognosis. J Pathol 1999;188:378-81.

13. Eyzaguirre EJ, Miettinen M, Norris B, Gatalica Z. Different immunohistochemical patterns of Fhit protein expression in renal neoplasms. Mod Pathol 1999;12:979-83.

14. Sozzi G, Pastorino U, Moiraghi L, Tagliabue E, Pezella F, Ghirelli C, et al. Loss of FHIT function in lung cancer and preinvasive bronchial lesions. Cancer Res 1998;58:5032-7.

15. Connolly DC, Greenspan DL, Wu R, Ren X, Dunn RL, Shah $\mathrm{KV}$, et al. Loss of Fhit expression in invasive cervical carcinomas and intraepithelial lesions associated with invasive disease. Clin Cancer Res 2000;6:3503-10. 
16. Kisselev LL, Justensen J, Wolfson AD, Frolova LY. Diadenosine oligophosphates (ApnA), a novel class of signaling molecules? FEBS Lett 1998;427:157-63.

17. Huebner K, Croce CM. FRA3B and other common fragile sites: the weakest links. Nature Rev 2001;1:214-21.

18. Vartanian A, Prudovsky I, Suzuki H, Dal Pra I, Kisselev L. Opposite effects of cell differentiation and apoptosis on Ap3A/Ap4A ratio in human cell cultures. FEBS Lett 1997;415: $160-2$.

19. Dumon KR, Ishii H, Vecchione A, Trapasso F, Baldassarre G, Chakrani F, et al. Fragile histidine triad expression delays tumor development and induces apoptosis in human pancreatic cancer. Cancer Res 2001;61:4827-36.

20. Sard L, Accornero P, Tornielli S, Delia D, Bunone G, Campiglio $\mathrm{M}$, et al. The tumor-suppressor gene FHIT is involved in the regulation of apoptosis and in cell cycle control. Proc Natl Acad Sci U S A 1999;96:8489-92.

21. Lin J, Bingliang F, Yen N, Fong K, Minna JD, Roth JA. Induction of apoptosis and inhibition of tumorigenicity and tumor growth by adenovirus vector-mediated fragile histidine triad (FHIT) gene overexpression. Cancer Res 1999;59:3333-9.

22. Ishii H, Dumon KR, Vecchione A, Fong LYY, Baffa R, Huebner K, Croce C. Potential cancer therapy with the fragile histidine triad gene. Review of the preclinical studies. JAMA 2001;286:2441-9.

23. Siprashvili Z, Sozzi G, Barnes LD, McCue P, Robinson AK, Eryomin V, et al. Replacement of Fhit in cancer cells suppresses tumorigenicity. Proc Natl Acad Sci U S A 1997;94: 13771-6.

24. Dumon KR, Ishii H, Fong LYY, Zanesi N, Fidanza V, Mancini $\mathrm{R}$, et al. FHIT gene therapy prevents tumor development in Fhit-deficient mice. Proc Natl Acad Sci U S A 2001;98:334651 .
25. Gatalica Z, Lele SM, Rampy BA, Norris BA. The expression of Fhit is related inversely to disease progression in patients with breast carcinoma. Cancer 2000;88:1378-83.

26. Vogelstein B, Kinzler KW. The multistep nature of cancer. Trends Genet 1993;9:138-41.

27. Kraggerud SM, Åman P, Holm R, Stenwig AE, Fosså SD, Nesland JM et al. Alterations of the fragile histidine triad gene, FHIT, and its encoded products contribute to testicular germ cell tumorigenesis. Cancer Res 2002;62:512-7.

28. Murphy GA, Halliday D, McLennan AG. The Fhit tumor suppressor protein regulates the intracellular concentration of diadenosine triphosphate but not diadenosine tetraphosphate. Cancer Res 2000;60:2342-4.

29. Chaudhuri AR, Khan IA, Prasad V, Robinson AK, Ludueña RF, Barnes LD. The tumor suppressor protein Fhit. A novel interaction with tubulin. J Biol Chem 1999;274:24378-82.

30. Roz L, Gramegna M, Ishii H, Croce CM, Sozzi G. Restoration of fragile histidine triad (FHIT) expression induces apoptosis and suppresses tumorigenicity in lung and cervical cancer cell lines. Proc Natl Acad Sci U S A 2002;99:3615-20.

31. Tanaka H, Shimada Y, Harada H, Shinoda M, Hatooka S, Imamura M, et al. Methylation of the 5' CpG island of the FHIT gene is closely associated with transcriptional inactivation in esophageal squamous cell carcinomas. Cancer Res 1998;58:3429-34.

32. Sato H, Hiyama K, Ishioka S, Maeda H, Yamakido M. Alternative splicing, but allelic loss, of the FHIT gene increases with development of lung cancer. Int J Oncol 1999;15:81-8.

33. Bieche I, Latil A, Becette V, Liderau R. Study of Fhit transcripts in normal and malignant breast tissue. Genes Chromosomes Cancer 1998;23:292-9. 